\title{
Ex Situ and In Situ Neutron Imaging of Enzymatic Electrochemical Cells
}

E. E. Looneya,t, G. J. Nelsona, ${ }^{\text {, }}$, , Z. K. van Zandta, Y. Ulyanovab, S. Singhalb, L. J. Santodonatoc, and H. Z. Bilheux ${ }^{d}$

a Department of Mechanical and Aerospace Engineering, University of Alabama in Huntsville, Huntsville, AL, USA

b CFD Research Corporation, Huntsville, AL, USA

c Instrument and Source Division, Oak Ridge National Laboratory, Oak Ridge, TN, USA

d Chemical and Engineering Materials Division, Oak Ridge National Laboratory, Oak Ridge, TN, USA

†Equal Contribution, listed alphabetically

*Corresponding Author: george.nelson@uah.edu

\section{Abstract}

Neutron imaging provides a route for studying the effect of environmental conditions on enzymatic electrochemical cell (EEC) performance. Here, the application of 2D and 3D neutron imaging is demonstrated as a means to investigate effects of environmental conditions on EEC performance. Changes in aqueous solutions that are vital to the operation of EECs have been directly observed in real time using neutron radiography (NR). These changes include uptake of aqueous solutions in electrode materials and in situ observation of water content during discharge. The EEC structure has also been observed using ex situ neutron tomography. Initially samples constructed to simulate EEC geometry were imaged, and aqueous buffer solution uptake behavior was observed in carbon paper cell components. In follow-on studies neutron tomography was used to map regions of enzyme catalyst inks, defining active regions within the EEC electrode. The higher neutron attenuation of polymeric ink components enables this observation. Finally, in situ imaging of a prototype EEC pouch cell was performed using a standard chronoamperometry arrangement while performing real time neutron radiography. Neutron radiographs acquired during operation show a clear variation in 
transmission behavior, primarily due to drying. Together these studies demonstrate the applicability and attendant challenges of neutron imaging for the study of EEC performance and reliability.

Keywords: biofuel cell; enzymatic fuel cell; enzymatic battery; in situ neutron imaging; neutron tomography

\section{Introduction}

Enzymatic electrochemical cells (EECs) are unique electrochemical devices capable of converting common sugars, alcohols, and other fuels directly into electrical energy through electrochemical reactions catalyzed by naturally occurring enzymes [1]. EECs may be operated as fuel cells or as batteries. A continuous fuel flow is supplied from an external source for fuel cell operation, while a fixed quantity of fuel "charges" the EEC to create a battery. The porous anode contains fuel within the liquid solution, and enzymes act as a catalyst to enhance oxidation of the fuel. EECs often use an air-breathing cathode, where enzymes act as a catalyst for the oxygen reduction reaction. Electrons for these reactions are blocked by the electrolyte and pass through an external circuit.

A major distinction in EEC configurations can be drawn between mediated electron transfer (MET) and direct electron transfer (DET). Electrical connection between the electrode and the enzyme presents a challenge traditionally overcome through the use of MET to facilitate the movement of electrons between the enzyme and electrode. These mediators can increase electrical connection between enzyme and electrode facilitating better performance. Common mediators include organometallic complexes or organic dyes immobilized on the electrode [2]. 
Mediators may impede performance through thermodynamic loss, mediator desorption from electrodes, and a general loss of device simplicity [3]. DET presents an attractive alternative, and can be accomplished using a glucose oxidase (GOx) bioanode and biocathodes made of either laccases (LACs) or bilirubin oxidase (BOD) [2]. DET performs the same oxidation process with the enzymes' active site having sufficient electrical connection with the electrode without a mediator. However, the power outputs of DET cells may not be comparable to the performance of MET cells [3].

Increased attention has also been given to development of materials for EECs that improve the stability of power output [4]. This broad topic leads into specific research on immobilization techniques, methods to keep the enzymes in the correct orientation for electrical "connection". By immobilizing the enzymes, EEC lifetimes have increased to several weeks instead of only hours. These techniques have also increased stability of the cells, experiencing smaller drops in power density throughout operation. For example, encapsulation of the enzyme catalyst within ion conductive polymers has been demonstrated to extend EEC life under standard operating conditions [4,5]. Shah et al. provide a thorough review of the advancements in immobilization techniques [5].

A third significant research thrust is miniaturization of enzymatic fuel cells, which has gained interest as more applications for EECs became apparent. The low power densities and open circuit voltages (OCV) are suitable for biomedical devices, small consumer electronics, and low power defense applications. These needs are best met with nanoscale devices, and several laboratories have been pursuing this objective [6-13]. In pursuit of the best performance, research groups have tested several different anodic 
enzymes, cathodic enzymes, and materials with various immobilization techniques or miniaturization schemes [11].

Enzymatic batteries and fuel cells can support a number of applications including: consumer electronics; medical implants; remote monitoring and sensing; and powering of small unmanned systems [1,14]. However, EECs exhibit significant performance degradation when exposed to elevated temperatures, dry conditions, or changes in $\mathrm{pH}$ [1]. This degradation is largely related to breakdown of the biocatalyst and an attendant loss in electrochemical activity. The combined effects of temperature and humidity require greater understanding to advance the implementation of enzymatic electrochemical cells, particularly with respect to retention of aqueous solutions that maintain favorable $\mathrm{pH}$ conditions.

In the expanding field of EEC work, there is limited information on environmental testing and water management during operation. These are both essential factors in the future use of EECs in environments outside of laboratory conditions. Studies of EECs operating on common sugars report results for testing at either room temperature or physiological conditions $[3,8,11,15-22]$. In a few cases the effects of temperature $[18,21,22]$ and $\mathrm{pH}[3,17,18,20,22]$ are addressed. To date most applications of alcohols as fuels for EECs have focused on room temperatures at near neutral $\mathrm{pH}$ [23-27]. In some cases acidic and alkaline conditions have been tested [26,27]. Further investigation of the effects of environmental conditions is critical, particularly for future applications in remote sensing and unmanned systems.

Enzymes are chains of amino acids that form into complex structures through bending and warping when exposed to specific environments or other enzymes. When 
operating within ideal conditions including a delicate $\mathrm{pH}$ balance, amount of substrate, and temperature, enzymes function at peak performance. However, if these conditions become unfavorable, the enzyme catalytic performance decreases, and in extreme conditions the enzymes denature and lose all functionality. The band of environmental conditions in which the enzymes within EECs can operate at peak performance is narrow, and the range in which operation is viable at lower performance is not completely understood.

Here, we report neutron radiography (NR) and tomography as a means of directly observing aqueous solution content within EECs and as a means of macroscopically mapping electroactive regions. Similar to x-ray radiography, a sample is placed in a neutron beam and attenuates (by scattering and absorption) neutrons at a rate corresponding to the materials present in the sample. The neutrons that transmit through the specimen impinge on a neutron converter screen (e.g. a scintillator) and create visible light photons that are then recorded as a 2D image using film or a charge coupled device (CCD). This neutron beam can be used to observe the interior of an object. When this process is performed for a single 2D image it is referred to as radiography. Neutron tomography can be performed by rotating the object in the neutron beam and acquiring a series of $2 \mathrm{D}$ images. These 2D images can be processed to provide a 3D image of the object.

A key difference between neutron and x-ray imaging is the type of materials that are amenable to imaging with each type of radiation. Generally, neutrons are better suited for observing materials based on light elements like hydrogen, lithium, or boron as well as some heavier elements like cadmium and gadolinium [28]. These materials attenuate neutrons and provide images with contrast that permits observation of detailed features. Conversely, x-rays are better suited for the observation of heavier elements, so NR 
provides a complement to x-ray techniques. Due to the high attenuation rates of hydrogen, imaging the content of water and other aqueous solutions within a sample is a useful application of this technique.

While application of neutron imaging on EEC water management has not been demonstrated, this technique has been applied to proton exchange membrane (PEM) fuel cells to good effect [28-32]. For example, Manke et al. [29] successfully utilized neutron radiography in conjunction with current density measurements in a PEM fuel cell to link water content with localized electrochemical performance within the cell. The limitations of a PEM cell with improper water management optimization were demonstrated, and relevant material choice and cell design issues were investigated. Park et al. [32] also completed a study of water management and performance of PEM fuel cells using NR. By using neutron imaging this team was able to demonstrate that the accumulation of water during dynamic loading of the cell slows down the cell's reactions considerably especially at high current density. Using the data from the neutron radiographs, a model for water accumulation prediction was successfully created. This method is ripe for use in EEC research due to the similarities in the structure and electrochemical processes of PEM fuel cells and EECs.

When determining the viability of EECs in conditions outside of the lab, a major concern is the inability of these cells to perform when the appropriate water conditions are not maintained. Neutron imaging provides a viable approach to test the functioning of EEC water management. The aqueous fuel and buffer solutions attenuate neutrons, revealing the change in solution over time through extended imaging sessions. The current output of the cell can also be mapped to the changing conditions within the cell observed through the 
real-time NR. Not only can the changing water conditions of the cell be mapped to current output, but the change in attenuation rates may also be correlated to variations in glucose concentration within the working electrode. In this way, the chemical activity of the cell can be observed directly based on regions where glucose fuel is consumed.

The present work focuses on observation of aqueous solution content and enzyme containing inks within EECs components. First, the applicability of neutron imaging methods to EEC materials is demonstrated using in situ studies of wetting behavior in cell components. Ex situ characterization of catalyst containing regions based on tomographic imaging of carbon felt electrodes is then discussed. Finally, in situ neutron imaging is demonstrated using a prototype EEC pouch cell. Together these studies demonstrate the applicability and attendant challenges of neutron imaging for the study of EEC performance and reliability.

\section{Experimental Methods}

\subsection{Electrode and Cell Preparation}

Initial model cells were constructed for proof-of-concept experiments to test the applicability of neutron imaging to EEC components and reactant solutions. These model cells simulated a membrane-separated EEC geometry as shown in Figure 1a, and were constructed from combinations of untreated carbon paper $(\sim 200 \mu \mathrm{m}$ thick $)$ and Bucky paper "electrodes" that bounded a Nafion separator. One region of carbon paper was kept open to the air, while the other was contained within a Teflon sealing gasket. This arrangement was intended to simulate an air breathing cathode EEC. The model cell components were held together between $\mathrm{Al}$ cover plates secured with $\mathrm{Al}$ tape. Aluminum was selected due to its neutron transparency and radioactive decay characteristics, which 
allow for relatively prompt changing of samples after imaging. An aqueous sodium phosphate buffer solution was used to wet the sealed carbon paper "anode" and preliminary radiographs were obtained for a set of 16 samples using either carbon paper or Buckypaper as the air side "cathode." Two types of separators were considered: Nafion protonated by boiling in sulfuric acid followed by washing and untreated Nafion. Variation in the Nafion treatment was included to test the neutron attenuation of the polymer electrolyte and the influence of pretreatment on solution retention. Combinations of water and buffer/fuel solution were also compared. Following initial NR measurements, buffer solution wicking was observed. One end of the model EEC was dipped into a buffer solution and the advancement of the solution was observed. A normalized image taken from this series, Figure $1 \mathrm{~b}$, demonstrates the capability to observe regions of buffer solution in the composite structure.

In situ imaging of an EEC during discharge was completed using a model enzymatic battery constructed in a three-electrode cell arrangement. The working electrode was made of carbon felt (CF) (approximately $6.35 \mathrm{~mm} \times 6.35 \mathrm{~mm} \times 25 \mathrm{~mm}$ ) containing an enzymatic ink (CFD Research Corporation, Huntsville, AL). This carbon felt (CF) material of $1.27 \mathrm{~cm}$ thickness (99\% purity) was acquired from Alfa Aesar (Ward Hill, MA) and cut into $15-20 \mathrm{~cm}$ strips with a thickness of $6.35 \mathrm{~mm}$. Next the anode was plasma treated for 5 minutes. Glucose Oxidase (GOx)-based ink was prepared by employing CFDRC proprietary carbon nanotube (CNT) ink formulation mixed with enzyme, such that the GOx loading was $37.8 \mathrm{mg} / \mathrm{mL}$. Key details of the performance and stability of this ink formulation have been outlined by Svoboda [33]. The enzymatic ink was gently vortexed for several minutes to facilitate complete mixing of all components. A total volume of $0.15 \mathrm{~mL}$ was casted evenly 
over the entire surface of each CF strip electrode, yielding an effective loading of $5.67 \mathrm{mg}$ of GOx on the electrode surface. Enzymatic electrodes were stored at $4^{\circ} \mathrm{C}$ for a minimum of 12 hours and allowed to equilibrate to ambient temperature prior to use. The counter and reference electrodes were bare carbon felt (approximately $9.5 \mathrm{~mm} \times 6.35 \mathrm{~mm}$ x $35 \mathrm{~mm}$ ) and silver paint on a carbon yarn $(\sim 90 \mathrm{mV}$ vs. $\mathrm{Ag} / \mathrm{AgCl})$, respectively. The electrodes were separated by filter paper and the entire structure was saturated with a $0.1 \mathrm{M}$ glucose fuel solution (0.1 M Glucose + 0.01 M Hydroquinone).

To assemble the EEC pouch cell two pieces of filter paper were placed around a reference electrode made of carbon thread and silver ink obtained from Alfa Aesar (Ward Hill, MA) and Ted Pella, Inc. (Redding, CA). The reference electrode structure was then taped to a Teflon support. A pre-wetted working electrode and counter electrode were then placed on top of the filter paper/Teflon assembly. Aluminum current collectors were placed on each of the electrode. Aluminum tape was placed over the current collector to secure the electrodes to the filter paper/Teflon assembly and mitigate evaporation of solution. The final product of this process was a three-electrode EEC pouch cell, illustrated in Figure 2.

\subsection{Electrochemical Characterization}

To characterize cell behavior prior to neutron imaging cyclic voltammetry (CV) and chronoamperometry tests were performed on EEC prototypes at UAH using a potentiostat/galvanostat (Bio-Logic VSP). This testing allowed for the assessment of cell materials, cell architecture, and provided insight into cell electrochemical response during the in situ discharge process. In these preliminary tests CV was performed using an $\mathrm{Ag} / \mathrm{AgCl}$ reference electrode with a scan rate of $100 \mathrm{mV} / \mathrm{s}$ between the voltages of $-0.8 \mathrm{~V}$ to 
$0.8 \mathrm{~V}$ vs. $\mathrm{Ag} / \mathrm{AgCl}$. Chronoamperometry was performed using several voltage steps and served as means of estimating discharge time for in situ experiments. During prototype cell development voltage steps of $0.5 \mathrm{~V}$ and $-0.3 \mathrm{~V}$ were applied to test oxidation and reduction behavior. For the in situ the neutron radiography experiments, the Ag on carbon yarn electrode noted above was applied as a reference electrode. A voltage of $-0.21 \mathrm{~V}$ vs. $\mathrm{Ag} / \mathrm{AgCl}$ provided consistent current and was used as a means of observing device activity under operational conditions.

\subsection{Neutron Imaging}

All neutron radiography and tomography measurements were performed using neutron imaging CG-1D facility at the ORNL High Flux Isotope Reactor. To ensure stability of the enzyme catalyst the electrodes were transported on ice in a cooler and kept under refrigeration prior to testing. The electrodes were allowed to equilibrate with ambient temperature for testing. The CG-1D beamline employs neutron radiation between 0.8 and 6 $\AA$ with a peak at $\sim 2.6 \AA$ as the probing radiation for imaging. Initial radiography measurements and water uptake observations were performed using a micro channel plate (MCP) detector. The MCP has a $28 \mathrm{~mm} \times 28 \mathrm{~mm}$ field of view. The neutron detection is equipped with a ${ }^{10} \mathrm{~B}-$ doped micro channel backed up with two MCPs made of glass material. Medipix2 is the readout system that is used on this detector [34]. The MCP detector has a resolution of $\sim 100 \mu \mathrm{m}$ with a pixel size of $\sim 55 \mu \mathrm{m}$. In subsequent tomography and in situ discharge measurements the detector used was a LiF/ZnS scintillator combined with a charge coupled device (CCD) camera. The field of view in this arrangement is $7.4 \mathrm{~cm} \mathrm{x} 7.4$ $\mathrm{cm}$. The CCD detector has a resolution of $\sim 100 \mu \mathrm{m}$ with a pixel size of $\sim 36 \mu \mathrm{m}$ at the sample position. 
Ex situ neutron tomography was performed on a set electrode materials to observe attenuation behavior prior to in situ imaging. Dry carbon felt, electrodes treated with enzyme catalyst ink, and electrodes that had been tested away from the beamline were imaged in these tests. Due to the relatively low attenuation of the carbon felt 10 second exposures were taken for the dry electrode samples. Images were taken of each specimen in $0.25^{\circ}$ angular increments from $0^{\circ}$ to $180^{\circ}$ of rotation. Tomographic imaging was also performed on an EEC pouch cell following in situ imaging during discharge. For the EEC pouch 60 second exposures were taken of each specimen in $0.5^{\circ}$ angular increments from $0^{\circ}$ to $180^{\circ}$ of rotation. These settings were chosen due to the higher neutron attenuation of the samples when saturated with the aqueous fuel and buffer solution. Tomographic data was reconstructed using TXM Wizard [35], which permitted realignment and iterative reconstruction capabilities for the parallel beam data obtained. The i-ART method [35], a form of iterative reconstruction, was found to improve reconstructed image quality compared to filtered back projection.

In situ radiography was first performed to observe buffer solution uptake in a model EEC, Figure 3. These measurements provided an initial confirmation that EEC components containing aqueous buffer solutions were amenable to neutron imaging. For these measurements a series of radiographs was taken over a 14 hour period using 60 second exposures. Data obtained from these measurements was processed using FIJI [36,37] and custom MATLAB (Mathworks, Natick, MA) scripts. A model enzymatic battery was constructed in a three electrode cell arrangement. A carbon felt working electrode containing an enzyme catalyst ink was applied. The counter and reference electrodes were carbon felt and $\mathrm{Ag} / \mathrm{AgCl}$, respectively. The electrodes were separated by filter paper and 
the entire structure was saturated with a $0.1 \mathrm{M}$ glucose solution in a sodium phosphate buffer. The cell was discharged using a standard chronoamperometry arrangement, stepping voltage to $-0.21 \mathrm{~V}$ vs. $\mathrm{Ag} / \mathrm{AgCl}$ and recording the resulting current. The potentiostat/galvanostat used for previous testing was used for in situ neutron imaging. Neutron radiographs were acquired at 60 second intervals during the discharge process. Images were acquired for approximately 5 hours, after which the cell was disconnected from the potentiostat/galvanostat. Following in situ testing tomographic imaging of the cell was performed, as noted above.

\section{Results and Discussion}

\subsection{Electrode Wetting Measurements}

Initial radiography measurements were performed using 16 samples constructed as shown in Figure 1. Minor variations in the solution retention characteristics of these samples were observed. For these test samples, leakage of buffer solution due to insufficient sealing was the main influencing factor on retention of the wetting solutions. Primarily, the carbon and Nafion components proved to be relatively transparent to the neutron radiation. A clear distinction was seen in the neutron attenuation by the aqueous solutions, as shown in Figure 1b.

Given the capability to distinguish regions of aqueous buffer and fuel solutions within the cell, a solution wetting experiment was performed. A sample constructed using carbon paper on the open air "cathode" side was used. The sample was placed vertically in a reservoir containing the aqueous buffer solution and a series of radiographs was taken over a period of 14 hours. The hydrophobicity of the carbon paper contributed to the gradual wetting behavior of the sample. The imaging data from the uptake experiment was 
normalized based on dark field and open beam images. The images were subsequently smoothed using $3 \times 3$ mean filter in FIJI. To highlight the wetted areas a moderate threshold of $\sim 0.97$ was applied to the normalized data. The output of the threshold operation was kept in a 32-bit image format to preserve the normalized attenuation of regions below the threshold that contained more of the aqueous buffer solution. The resulting images were plotted as color contour plots using MATLAB to provide a false color representation of the wet and dry areas within the sample. Preliminary observation of progressive sample saturation, Figure 3, suggests absorption data can provide insight into the dynamics of fuel and buffer solution retention within the electrodes. Blue regions, corresponding to increased neutron attenuation, indicate areas with higher water content, while red regions with lower attenuation indicate dry areas of the sample. Due to imperfect sealing some significant wetting is seen at the edges of the model cell structure. These areas yield the highest attenuation. A more gradual wetting of the carbon paper can be seen progressing from the lower left of the sample as pores within the membrane fill with the buffer solution.

\subsection{Ex Situ Tomography of Dry Electrodes}

Following successful demonstration of neutron imaging for observation of aqueous solution uptake in EEC components in situ imaging of an enzymatic battery was pursued. As a first step, neutron tomography was used to observe the structure of the carbon felt electrodes used in the EEC pouch cells. Eight samples were imaged: one unloaded electrode taken from an operated EEC, one unloaded unused electrode, three used electrodes that had been loaded with enzymatic ink, and three unused electrodes that had been loaded with enzymatic ink. This measurement was done to determine the attenuation 
characteristics of the carbon felt. However, an initially unexpected capability found in this measurement was the capability to map regions of enzyme catalyst inks, defining active regions within the EEC electrode. An absorption based radiograph of the electrodes imaged in these measurements is shown in Figure 4a along with a key indicating the type of electrode at each position in the radiograph. Enzyme catalyst regions are shown in 3D isosurface renderings for an unused electrode (Figure 4b) and a used electrode (Figure 4c). The higher neutron attenuation of polymeric ink components enables this observation. Loss of the catalyst ink can be observed in the samples imaged after operation. Some redistribution of the material is also observed, as indicated by the 3D isosurface renderings of the used electrode. The case shown in Figure 4c is an example of significant catalyst ink loss. The observed loss may occur either during the process of testing, the subsequent drying of the electrodes, or both processes. Deposits with higher attenuation are seen within the used counter electrode, as seen in Figure 4a. These deposits may be catalyst ink or crystalized glucose from fuel. However, no clear evidence of fuel crystallization was observed when inspecting the used counter electrode.

Using a basic voxel counting approach, the volume of the catalyst ink regions is estimated to be reduced from an average of $\sim 0.042 \mathrm{~mL}$ for the fresh electrodes to $\sim 0.017$ $\mathrm{mL}$ for the used electrodes. Here it is important to note that this comparison is not a direct before and after comparison of the same electrode. However, the unused and used electrodes were taken from the same piece of treated carbon felt. Furthermore, the catalyst loading is underestimated due to the limited resolution of the NR detector applied. It is likely regions containing biocatalyst ink small volumes are not discernible, due to a lack of total attenuation. Loss of ink during transport and handling may also contribute to this 
discrepancy. Complementary imaging using either x-ray microtomography or a higher resolution NR detector would mitigate this underestimation. In the present work, the volume comparison may be treated as a qualitative assessment of catalyst loss that might occur during operation. The capability to observe enzyme containing regions could be quite useful in the macroscopic design and development of future EECs. Observing catalytic regions and the related performance effects could better inform development of processes for infiltration of enzymatic inks within the electrodes so that increased electrode activity may be achieved. Furthermore, documenting active regions within test cells can aid in connecting the effects of drying to overall cell performance.

\subsection{In Situ EEC Neutron Imaging}

The capability to observe aqueous fuel and buffer solutions within EEC electrodes makes them candidates for study using neutron radiography and tomography. This capability has been demonstrated above for both ex situ radiography and tomography as well as in situ studies of aqueous solution wetting behavior. In situ neutron radiography is demonstrated here for a prototype EEC pouch cell. The current generated during this test is shown in Figure 5, along with representative CV scans from two prototype cells (inset). The total charge transfer during the in situ test is on the order of 3-4\% of total amount of glucose fuel contained in both electrodes. Further cell characterization is needed to establish fuel loading and voltage settings that will produce more significant consumption of the reactant species. For the present studies, the drying behavior of the cell was found to be a more significant factor. However, preliminary investigations suggest that glucose concentration can be tracked based on neutron attenuation characteristics. 
Figure $6 a$ shows a transmission based neutron radiograph of the test cell, highlighting the carbon felt working and counter electrodes, as well as the filter paper separator with reference electrode. Following the discharge measurement tomography was performed on the pouch cell revealing areas where the aqueous fuel/buffer solution was lost during discharge. A cross-section taken from the resulting tomogram is shown in Figure 6b. This cross-section is shown based on attenuation, with more saturated regions that have greater absorption appearing white. Drying is seen in portions of both electrodes but it is most substantial within the counter electrode.

The radiograph shown in Figure $6 a$ is one of a series of images taken every 60 s over the course of discharge. A region of interest (ROI, shown as the yellow box in Figure 6a) was selected based on more consistent saturation, as seen in both the $2 \mathrm{D}$ data and within the tomographic data, and the average intensity of transmitted neutrons for this region was assessed as a function of time and distance across the electrode ( $\mathrm{x}$ vector below the ROI in Figure 6a). The transmitted intensity data for the ROI is shown in Figure 6c, with clear variation in transmission behavior. The upward slope near the active region of the cell (region 1), on the left edge of the ROI, suggests a locally increased glucose concentration may be present as the result of reduction. It is important to note that other reactants and products may be present within this region, and further assessment of the local glucose concentration is needed. The general decrease in the intensity across the ROI (region 2) suggests reduced attenuation of the incident radiation that most likely associated with drying. At the right edge of the ROI (region 3) a significant decrease in intensity is seen. This decrease is due to faster solution loss near the current collector and some variation in transmission length at the edge of the sample caused by slight sample misalignment in the 
neutron beam. In general, drying holds greatest influence on changes in neutron transmission in the present studies. Even within the relatively saturated ROI signs of drying can be seen, as evinced by temporal variations in intensity seen in the area 2-3 $\mathrm{mm}$ across the electrode. These variations become more significant if the size of the ROI is expanded to capture areas near the top of the working electrode.

While these results demonstrate the capability for in situ studies, several sources of error must be addressed. First, better sealing of the EEC is required to reduce drying due to leakage. This sealing includes control of solution wicking into the filter paper regions, which may be achieved by implementing a separator. Second, total fuel loading and voltage settings must be further analyzed to better observe fuel consumption. As noted in these initial studies the cell was oversaturated with glucose, leading to a small change in fuel concentration during tests. Finally, review of the reconstructed tomogram revealed misalignment in the sample that contributes to a variation in the transmission length across the width of the radiographic data set. This variation was most significant in the areas containing the filter paper separators and Teflon support. The ROI shown in Figure 6 was selected such that it was not significantly affected by this variation in transmission length. Ultimately, better alignment of the cell is needed to control the transmission length through the working electrode and reduce the influence of geometric variations on the observed transmitted intensity. Development and characterization of test cells to mitigate these sources of error is underway.

\section{Conclusions}

Exposure to higher temperatures, lower humidity, or non-neutral $\mathrm{pH}$ can lead to performance loss in enzymatic electrochemical cells due to enzyme deactivation. Neutron 
imaging can serve as a tool for EEC development by permitting in situ observation of cells under operational conditions. To this end, neutron radiography and tomography have been demonstrated as a means of directly observing aqueous solution content within EECs and macroscopically mapping electroactive regions within the electrodes. Changes in aqueous solutions in EECs and their components have been directly observed in real time using 2D neutron radiography. These changes include uptake of aqueous solutions in electrode materials and in situ observation of water content during operation. Ex situ neutron tomography has also been applied to map regions of enzyme catalyst inks.

Initially samples constructed to simulate EEC geometry were imaged, and aqueous buffer solution uptake behavior was observed in carbon paper cell components. Absorption data suggested that water content of EEC materials can be assessed using neutron imaging. While uneven wetting arose due to imperfect sealing, gradual infiltration of an aqueous buffer solution within carbon cloth electrodes could be seen clearly.

The EEC structure has also been observed using ex situ neutron tomography to map regions of enzyme catalyst inks. The resulting image data was used to define active regions within the EEC electrode and compare the enzyme catalyst ink content of fresh and used electrodes from EEC tests. A loss of catalyst ink was observed for the working electrodes that had been operated, and a slight uptake of biocatalyst ink is suspected for the used counter electrode. While a more direct comparison of electrodes before and after use is needed, these results suggest that active regions can be tracked and analyzed using neutron techniques.

In situ observation of a prototype EEC pouch cell was also performed using real time NR over an extended time period. Neutron attenuation for the working electrode was 
averaged for a region of interest and plotted as function of time. The observed trends in the attenuation data support the occurrence of a reduction reaction. Higher attenuation, corresponding to increased glucose content, is seen around the active region within the cell. A downward slope toward the inactive regions of the cell suggests a flux away from the active region, against the concentration gradient. These results provide a preliminary demonstration of the capability for in situ neutron imaging studies of enzymatic batteries and fuel cells. However, significant regions of drying were observed in the prototype cells tested. While this observation further demonstrates the merit of neutron techniques for observing solution uptake and drying, better sealing and control of aqueous solutions is needed to provide clearer observations of reactions within the electrodes.

Taken together the above studies demonstrate that neutron radiography and tomography can be applied for the ex situ and in situ assessment of enzymatic electrochemical cells. Ex situ studies can be used to support cell design and development of fabrication methods, particularly with respect to techniques for loading porous electrodes with catalyst containing inks. In situ techniques provide a clear capability for observing solution uptake and drying behavior related to operational conditions. The results provided suggest that in situ observation of chemical activity can be achieved as well.

\section{Acknowledgments}

Support from a University of Alabama in Huntsville New Faculty Research grant is gratefully acknowledged. A portion of this research at ORNL's High Flux Isotope Reactor was sponsored by the Scientific User Facilities Division, Office of Basic Energy Sciences, U.S. Department of Energy. 


\section{References}

[1] S. Freguia, B. Virdis, F. Harnisch, J. Keller, Bioelectrochemical systems: Microbial versus enzymatic catalysis, Electrochimica Acta. 82 (2012) 165-174. doi:10.1016/j.electacta.2012.03.014.

[2] S.D. Minteer, B.Y. Liaw, M.J. Cooney, Enzyme-based biofuel cells, Curr. Opin. Biotechnol. 18 (2007) 228-234. doi:10.1016/j.copbio.2007.03.007.

[3] Y. Kamitaka, S. Tsujimura, N. Setoyama, T. Kajino, K. Kano, Fructose/dioxygen biofuel cell based on direct electron transfer-type bioelectrocatalysis, Phys. Chem. Chem. Phys. 9 (2007) 1793. doi:10.1039/b617650j.

[4] S.D. Minteer, P. Atanassov, H.R. Luckarift, G.R. Johnson, New materials for biological fuel cells, Mater. Today. 15 (2012) 166-173.

[5] M.H. Osman, A.A. Shah, F.C. Walsh, Recent progress and continuing challenges in biofuel cells. Part I: Enzymatic cells, Biosens. Bioelectron. 26 (2011) 3087-3102. doi:10.1016/j.bios.2011.01.004.

[6] M. Falk, Z. Blum, S. Shleev, Direct electron transfer based enzymatic fuel cells, Electrochimica Acta. 82 (2012) 191-202. doi:10.1016/j.electacta.2011.12.133.

[7] A. de Poulpiquet, A. Ciaccafava, E. Lojou, New trends in enzyme immobilization at nanostructured interfaces for efficient electrocatalysis in biofuel cells, Electrochimica Acta. 126 (2014) 104-114. doi:10.1016/j.electacta.2013.07.133.

[8] V. Andoralov, M. Falk, D.B. Suyatin, M. Granmo, J. Sotres, R. Ludwig, et al., Biofuel Cell Based on Microscale Nanostructured Electrodes with Inductive Coupling to Rat Brain Neurons, Sci. Rep. 3 (2013). doi:10.1038/srep03270. 
[9] C. Pan, Y. Fang, H. Wu, M. Ahmad, Z. Luo, Q. Li, et al., Generating Electricity from Biofluid with a Nanowire-Based Biofuel Cell for Self-Powered Nanodevices, Adv. Mater. 22 (2010) 5388-5392. doi:10.1002/adma.201002519.

[10] M. Togo, A. Takamura, T. Asai, H. Kaji, M. Nishizawa, Structural studies of enzymebased microfluidic biofuel cells, J. Power Sources. 178 (2008) 53-58. doi:10.1016/j.jpowsour.2007.12.052.

[11] M. Togo, A. Takamura, T. Asai, H. Kaji, M. Nishizawa, An enzyme-based microfluidic biofuel cell using vitamin K3-mediated glucose oxidation, Electrochimica Acta. 52 (2007) 4669-4674. doi:10.1016/j.electacta.2007.01.067.

[12] S. Calabrese Barton, J. Gallaway, P. Atanassov, Enzymatic Biofuel Cells for Implantable and Microscale Devices, Chem. Rev. 104 (2004) 4867-4886. doi:10.1021/cr020719k.

[13] K.G. Lim, G.T.R. Palmore, Microfluidic biofuel cells: The influence of electrode diffusion layer on performance, Biosens. Bioelectron. 22 (2007) 941-947. doi:10.1016/j.bios.2006.04.019.

[14] M.J. Moehlenbrock, S.D. Minteer, Extended lifetime biofuel cells, Chem. Soc. Rev. 37 (2008) 1188. doi:10.1039/b708013c.

[15] A. Zebda, C. Gondran, A. Le Goff, M. Holzinger, P. Cinquin, S. Cosnier, Mediatorless highpower glucose biofuel cells based on compressed carbon nanotube-enzyme electrodes, Nat. Commun. 2 (2011) 370. doi:10.1038/ncomms1365.

[16] C. Bunte, L. Hussein, G.A. Urban, Performance of non-compartmentalized enzymatic biofuel cell based on buckypaper cathode and ferrocene-containing redox polymer anode, J. Power Sources. 247 (2014) 579-586. doi:10.1016/j.jpowsour.2013.08.077. 
[17] K. Stolarczyk, M. Kizling, D. Majdecka, K. Żelechowska, J.F. Biernat, J. Rogalski, et al., Biobatteries and biofuel cells with biphenylated carbon nanotubes, J. Power Sources. 249 (2014) 263-269. doi:10.1016/j.jpowsour.2013.10.074.

[18] R.E. Kim, S.-G. Hong, S. Ha, J. Kim, Enzyme adsorption, precipitation and crosslinking of glucose oxidase and laccase on polyaniline nanofibers for highly stable enzymatic biofuel cells, Enzyme Microb. Technol. 66 (2014) 35-41. doi:10.1016/j.enzmictec.2014.08.001.

[19] V. Coman, R. Ludwig, W. Harreither, D. Haltrich, L. Gorton, T. Ruzgas, et al., A Direct Electron Transfer-Based Glucose/Oxygen Biofuel Cell Operating in Human Serum, Fuel Cells. (2009) NA-NA. doi:10.1002/fuce.200900121.

[20] V. Coman, C. Vaz-Domínguez, R. Ludwig, W. Harreither, D. Haltrich, A.L. De Lacey, et al., A membrane-, mediator-, cofactor-less glucose/oxygen biofuel cell, Phys. Chem. Chem. Phys. 10 (2008) 6093. doi:10.1039/b808859d.

[21] Z. Zhu, F. Sun, X. Zhang, Y.-H.P. Zhang, Deep oxidation of glucose in enzymatic fuel cells through a synthetic enzymatic pathway containing a cascade of two thermostable dehydrogenases, Biosens. Bioelectron. 36 (2012) 110 - 115. doi:http://dx.doi.org/10.1016/j.bios.2012.04.001.

[22] X. Wu, F. Zhao, J.R. Varcoe, A.E. Thumser, C. Avignone-Rossa, R.C.T. Slade, A onecompartment fructose/air biological fuel cell based on direct electron transfer, Biosens. Bioelectron. 25 (2009) 326-331. doi:10.1016/j.bios.2009.07.011.

[23] N.L. Akers, C.M. Moore, S.D. Minteer, Development of alcohol/O2 biofuel cells using salt-extracted tetrabutylammonium bromide/Nafion membranes to immobilize 
dehydrogenase enzymes, Electrochimica Acta. 50 (2005) 2521-2525.

doi:10.1016/j.electacta.2004.10.080.

[24] S. Topcagic, S.D. Minteer, Development of a membraneless ethanol/oxygen biofuel cell, Electrochimica Acta. 51 (2006) 2168-2172. doi:10.1016/j.electacta.2005.03.090.

[25] R.L. Arechederra, B.L. Treu, S.D. Minteer, Development of glycerol/02 biofuel cell, J. Power Sources. 173 (2007) 156-161. doi:10.1016/j.jpowsour.2007.08.012.

[26] A. Ramanavicius, A. Kausaite, A. Ramanaviciene, Enzymatic biofuel cell based on anode and cathode powered by ethanol, Biosens. Bioelectron. 24 (2008) 761-766. doi:10.1016/j.bios.2008.06.048.

[27] X.-C. Zhang, A. Ranta, A. Halme, Direct methanol biocatalytic fuel cell—Considerations of restraints on electron transfer, Biosens. Bioelectron. 21 (2006) 2052-2057. doi:10.1016/j.bios.2006.01.035.

[28] M. Strobl, I. Manke, N. Kardjilov, A. Hilger, M. Dawson, J. Banhart, Advances in neutron radiography and tomography, J. Phys. Appl. Phys. 42 (2009) 243001. doi:10.1088/0022-3727/42/24/243001.

[29] I. Manke, H. Markötter, C. Tötzke, N. Kardjilov, R. Grothausmann, M. Dawson, et al., Investigation of Energy-Relevant Materials with Synchrotron X-Rays and Neutrons, Adv. Eng. Mater. 13 (2011) 712-729.

[30] A.Z. Weber, M.A. Hickner, Modeling and high-resolution-imaging studies of watercontent profiles in a polymer-electrolyte-fuel-cell membrane-electrode assembly, Electrochimica Acta. 53 (2008) 7668-7674.

[31] K.T. Cho, A. Turhan, J.H. Lee, J.S. Brenizer, A.K. Heller, L. Shi, et al., Probing water transport in polymer electrolyte fuel cells with neutron radiography, Nucl. Instrum. 
Methods Phys. Res. Sect. Accel. Spectrometers Detect. Assoc. Equip. 605 (2009) 119122.

[32] J. Park, X. Li, D. Tran, T. Abdel-Baset, D.S. Hussey, D.L. Jacobson, et al., Neutron imaging investigation of liquid water distribution in and the performance of a $\{\mathrm{PEM}\}$ fuel cell, Int. J. Hydrog. Energy. 33 (2008) 3373 - 3384.

doi:http://dx.doi.org/10.1016/j.jhydene.2008.03.019.

[33] V. Svoboda, J. Wei, S. Singhal, Y. Ulyanova, Electrically Conductive Ink and Uses Thereof, US20140183416 A1, 2014.

[34] A.S. Tremsin, J.B. McPhate, J.V. Vallerga, O.H.W. Siegmund, J.S. Hull, W.B. Feller, et al., Detection efficiency, spatial and timing resolution of thermal and cold neutron counting MCP detectors, Nucl. Instrum. Methods Phys. Res. Sect. Accel. Spectrometers Detect. Assoc. Equip. 604 (2009) 140-143. doi:10.1016/j.nima.2009.01.041.

[35] Y.J. Liu, P.P. Zhu, B. Chen, J.Y. Wang, Q.X. Yuan, W.X. Huang, et al., A new iterative algorithm to reconstruct the refractive index, Phys. Med. Biol. 52 (2007) L5-L13.

[36] J. Schindelin, I. Arganda-Carreras, E. Frise, V. Kaynig, M. Longair, T. Pietzsch, et al., Fiji: an open-source platform for biological-image analysis, Nat Meth. 9 (2012) 676-682. doi:10.1038/nmeth.2019.

[37] C.A. Schneider, W.S. Rasband, K.W. Eliceiri, NIH Image to ImageJ: 25 years of image analysis, Nat Meth. 9 (2012) 671-675. doi:10.1038/nmeth.2089. 


\section{Figure Captions}

Figure 1. (a.) A model EEC composed of carbon paper electrodes and a Nafion separator was used to observe saturation behavior. (b.) A neutron radiograph shows distinct regions where the aqueous buffer solution wets the porous electrodes.

Figure 2. Schematic of the prototype EEC pouch cell used for in situ neutron imaging. For the present studies filter paper was used as a separator between the electrodes. A piece of Teflon gasket material was used as non-conductive support for the cell.

Figure 3. Neutron transmission radiographs of the saturation of electrode materials used in EECs. Blue regions represent greater content of aqueous fuel and buffer solutions. Red indicates dry regions in the sample.

Figure 4. (a.) Neutron radiograph taken from a tomographic imaging series for enzymatic electrochemical cell electrodes, shown in absorption mode with higher attenuation regions indicated in white. Used and unused electrodes as well as electrodes containing enzymatic ink and untreated electrodes were compared. The electrodes are labeled accordingly to the right of the radiograph. Isosurface renderings of tomography data enable comparison of catalyst regions from (b.) unused and (c.) used EEC electrodes (samples 6 and 3, respectively) indicate the loss of enzyme containing ink for some electrodes. The images at the top in (b.) and (c.) are 3D views of the catalyst region. Top down views are show below these images. The grid spacing shown is approximately $2 \mathrm{~mm}$ for the $3 \mathrm{D}$ plots. 
Figure 5. Current generated during in situ imaging of enzymatic battery imaging shows a reduction reaction with relatively low charge transfer compared to the total glucose loading of the cell (3-4\% of total loading). Chronoamperometry was performed by stepping the cell to a voltage of $-0.21 \mathrm{~V}$ vs. $\mathrm{Ag} / \mathrm{AgCl}$. Representative cyclic voltammetry results for a prototype cell are shown in the inset.

Figure 6. (a.) Transmission radiograph of test cell structure at time $t=60 \mathrm{~s}$. The image has been filtered to remove $\mathrm{Al}$ tape and current collectors. A region of interest with consistent saturation is indicated in yellow. A $5 \mathrm{~mm}$ scale bar is shown at the lower left. (b.) A crosssection of the EEC pouch cell taken from attenuation-based reconstructed tomographic data reveals regions where drying of the electrodes has occurred, as indicated by the dark regions within the working and counter electrodes. (c.) Relative absorption behavior indicates a gradual change in solution content due to combined effects of reaction and electrode drying. Three regions are seen corresponding to the active region (1), the bulk electrode (2), and the edge of the electrode near the current collector (3). 

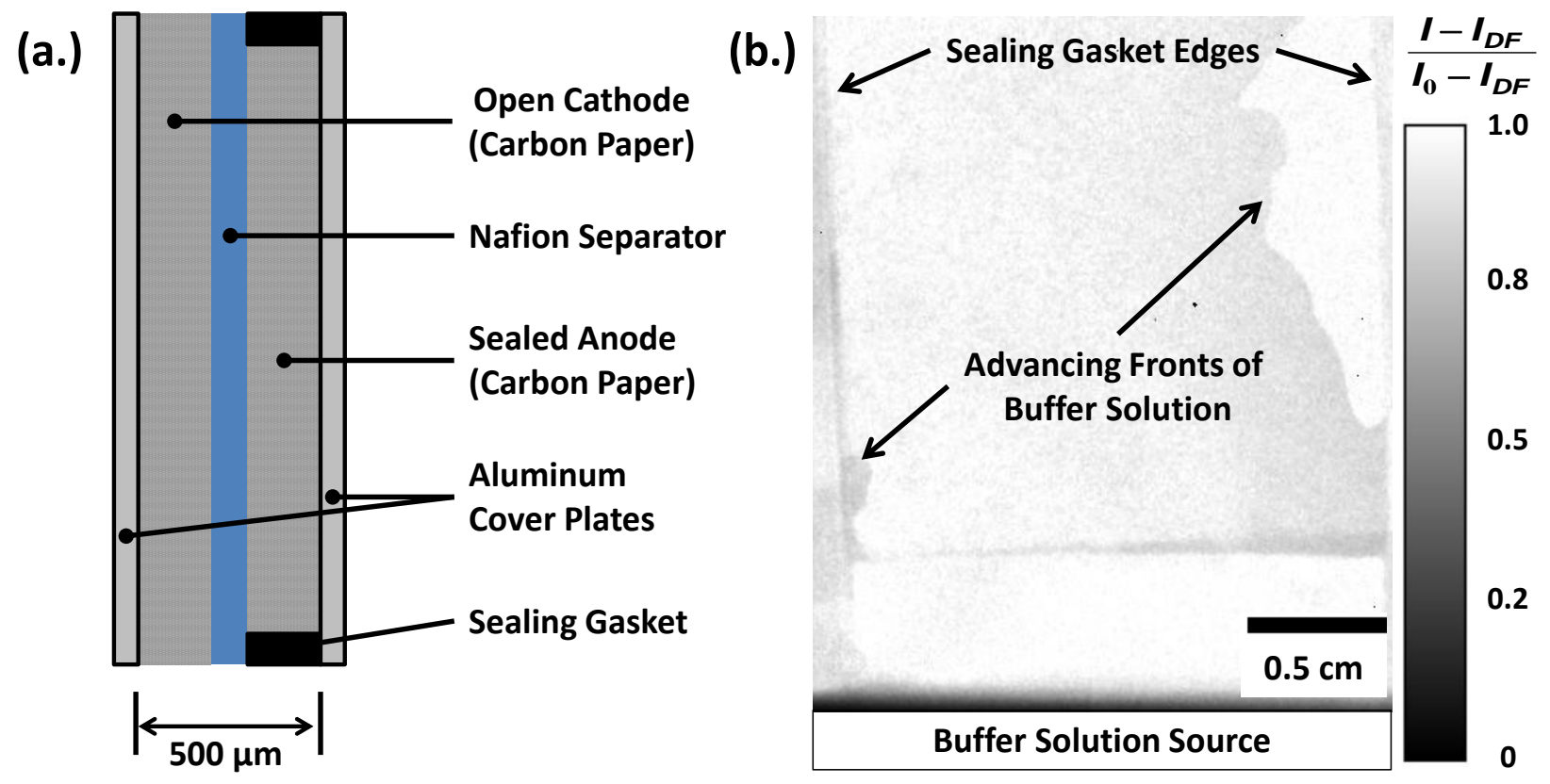

Figure 1. (a.) A model EEC composed of carbon paper electrodes and a Nafion separator was used to observe saturation behavior. (b.) A neutron radiograph shows distinct regions where the aqueous buffer solution wets the porous electrodes. 


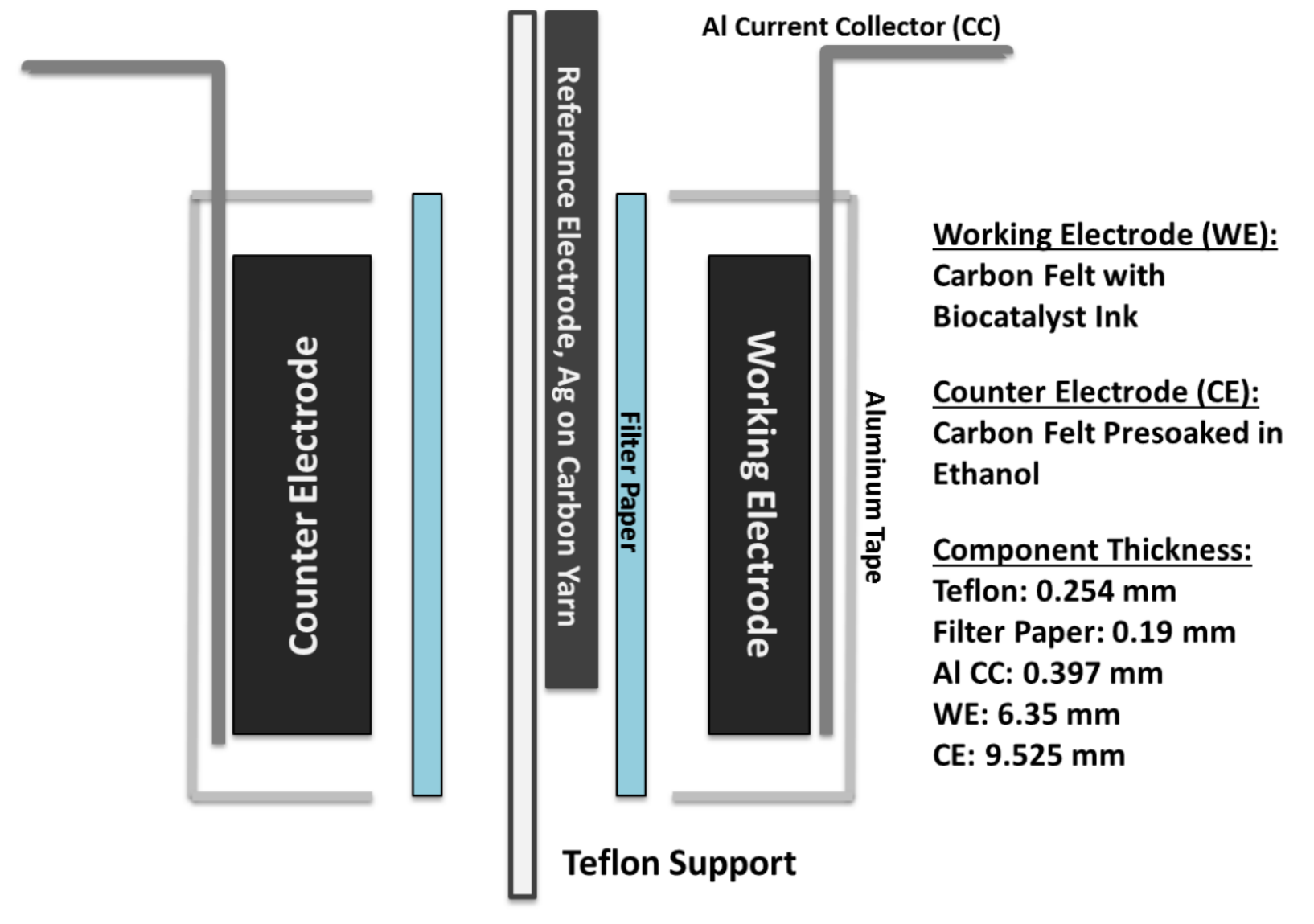

Figure 2. Schematic of the prototype EEC pouch cell used for in situ neutron imaging. For the present studies filter paper was used as a separator between the electrodes. A piece of Teflon gasket material was used as non-conductive support for the cell. 

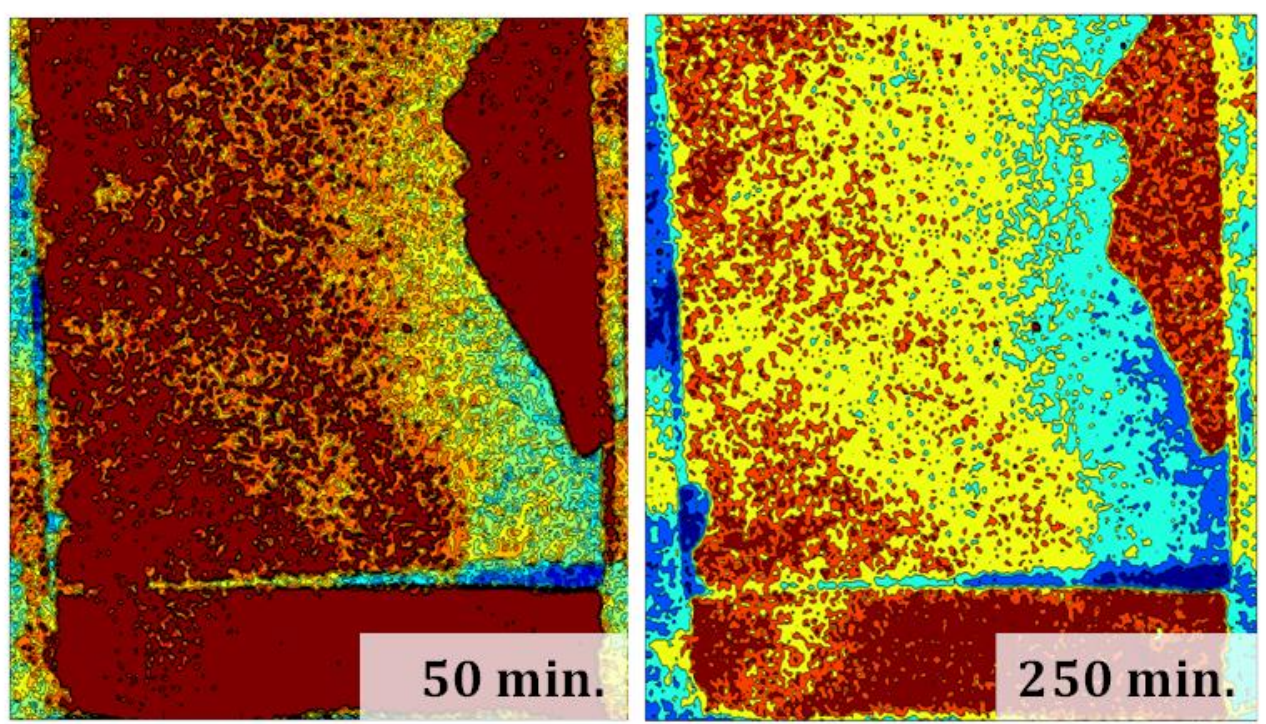

$\frac{I-I_{D F}}{I_{0}-I_{D F}}$
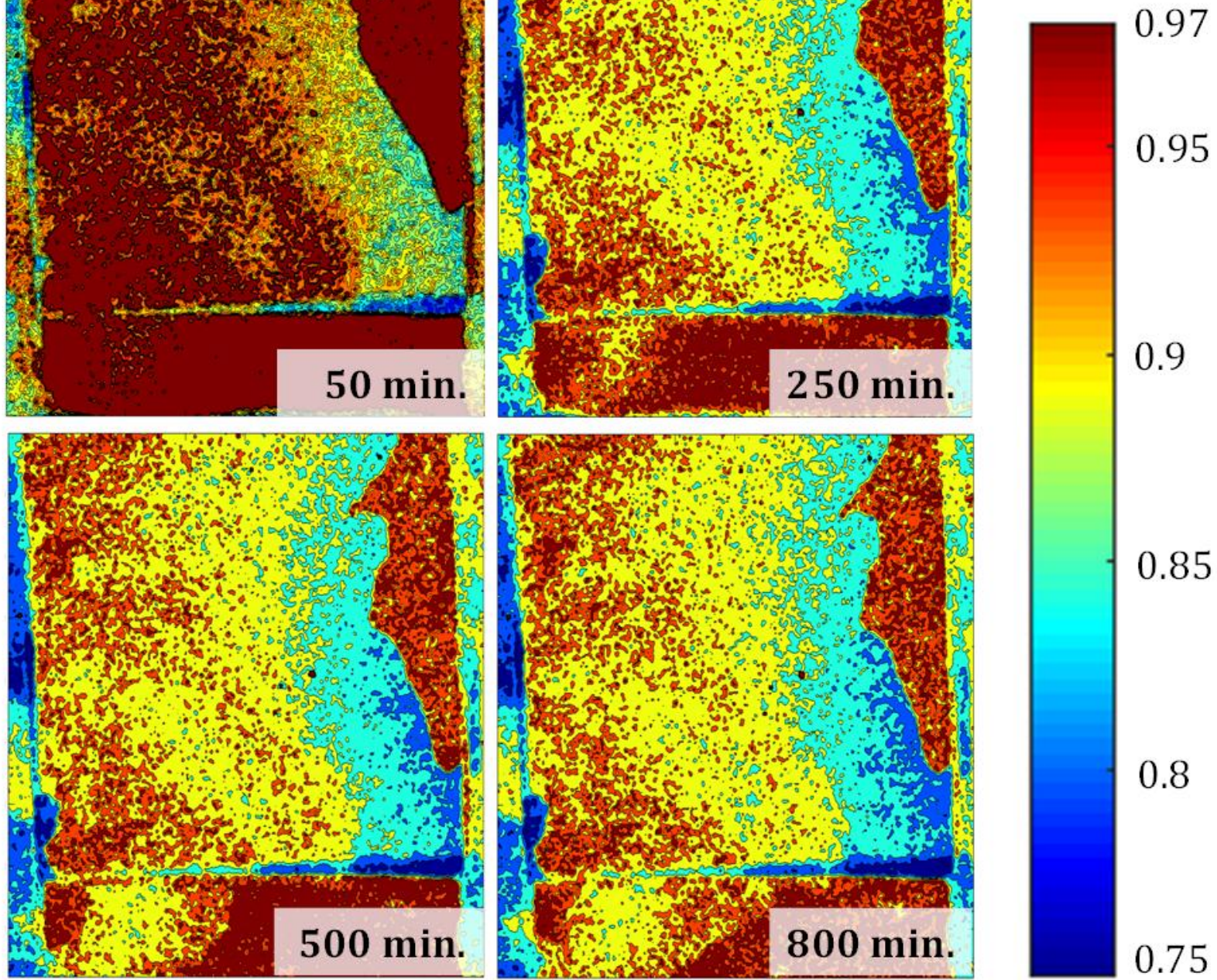

Figure 3. Neutron transmission radiographs of the saturation of electrode materials used in EECs. Blue regions represent greater content of aqueous fuel and buffer solutions. Red indicates dry regions in the sample. 

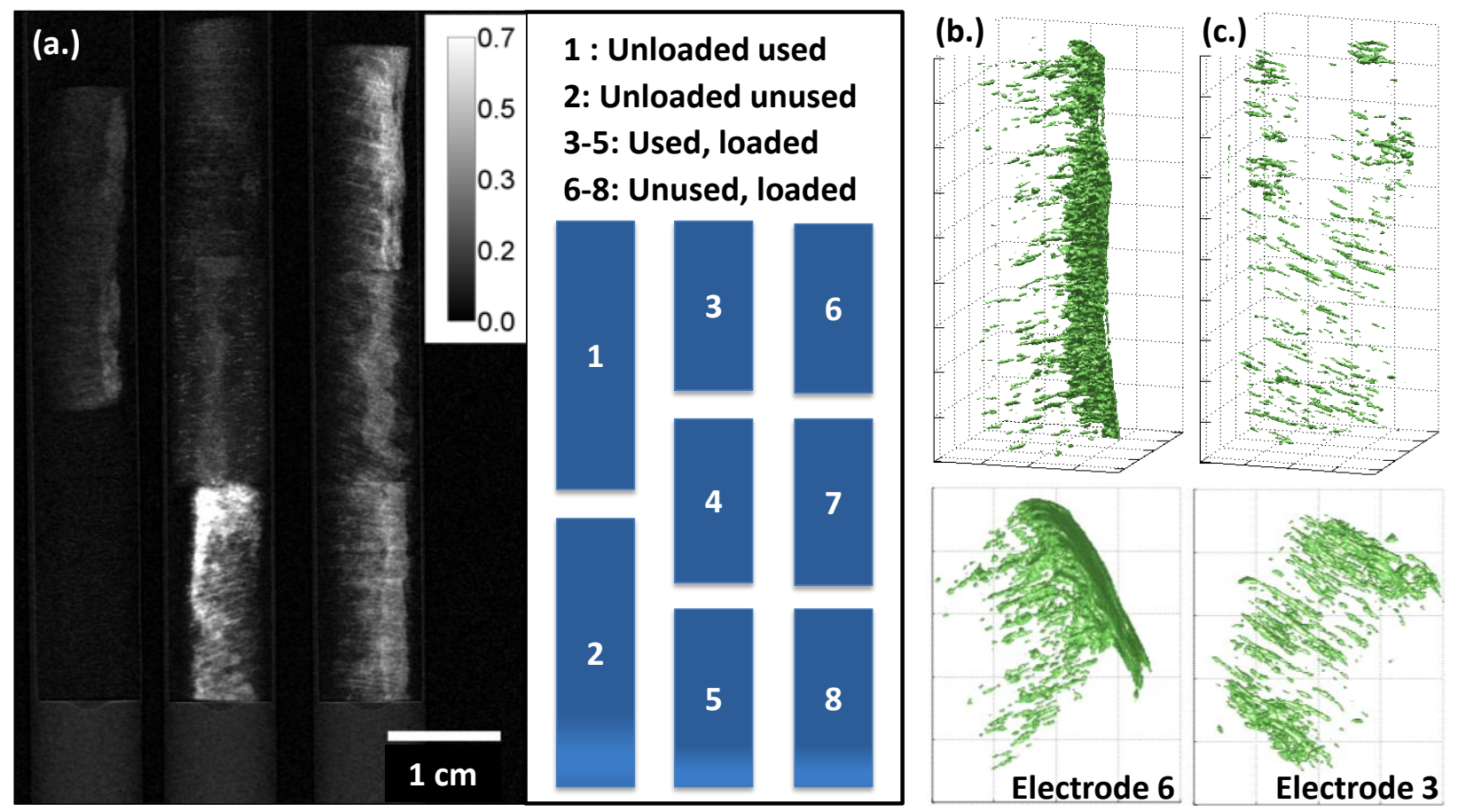

Figure 4. (a.) Neutron radiograph taken from a tomographic imaging series for enzymatic electrochemical cell electrodes, shown in absorption mode with higher attenuation regions indicated in white. Used and unused electrodes as well as electrodes containing enzymatic ink and untreated electrodes were compared. The electrodes are labeled accordingly to the right of the radiograph. Isosurface renderings of tomography data enable comparison of catalyst regions from (b.) unused and (c.) used EEC electrodes (samples 6 and 3, respectively) indicate the loss of enzyme containing ink for some electrodes. The images at the top in (b.) and (c.) are 3D views of the catalyst region. Top down views are show below these images. The grid spacing shown is approximately $2 \mathrm{~mm}$ for the $3 \mathrm{D}$ plots. 


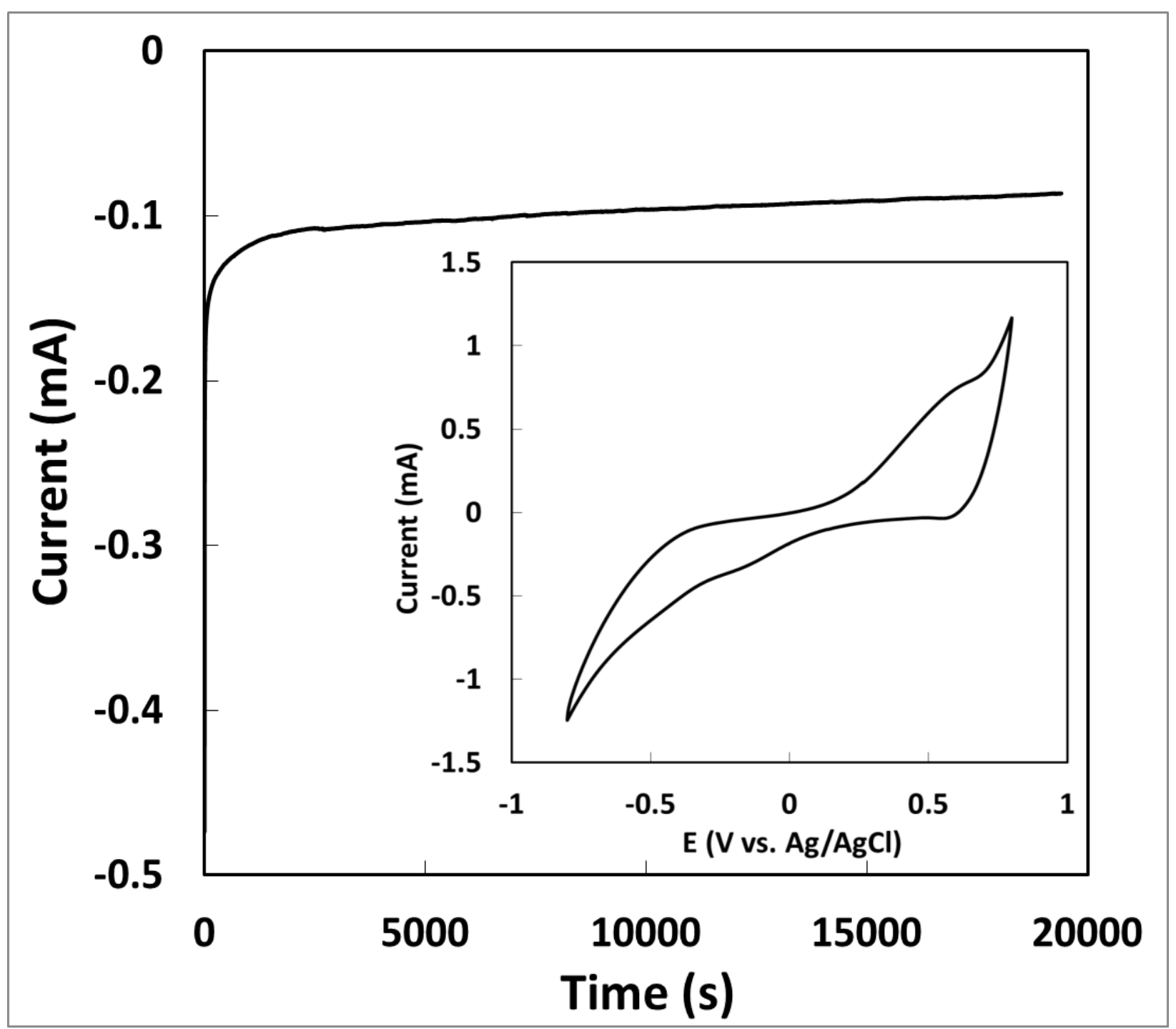

Figure 5. Current generated during in situ imaging of enzymatic battery imaging shows a reduction reaction with relatively low charge transfer compared to the total glucose loading of the cell (3-4\% of total loading). Chronoamperometry was performed by stepping the cell to a voltage of $-0.21 \mathrm{~V}$ vs. $\mathrm{Ag} / \mathrm{AgCl}$. Representative cyclic voltammetry results for a prototype cell are shown in the inset. 

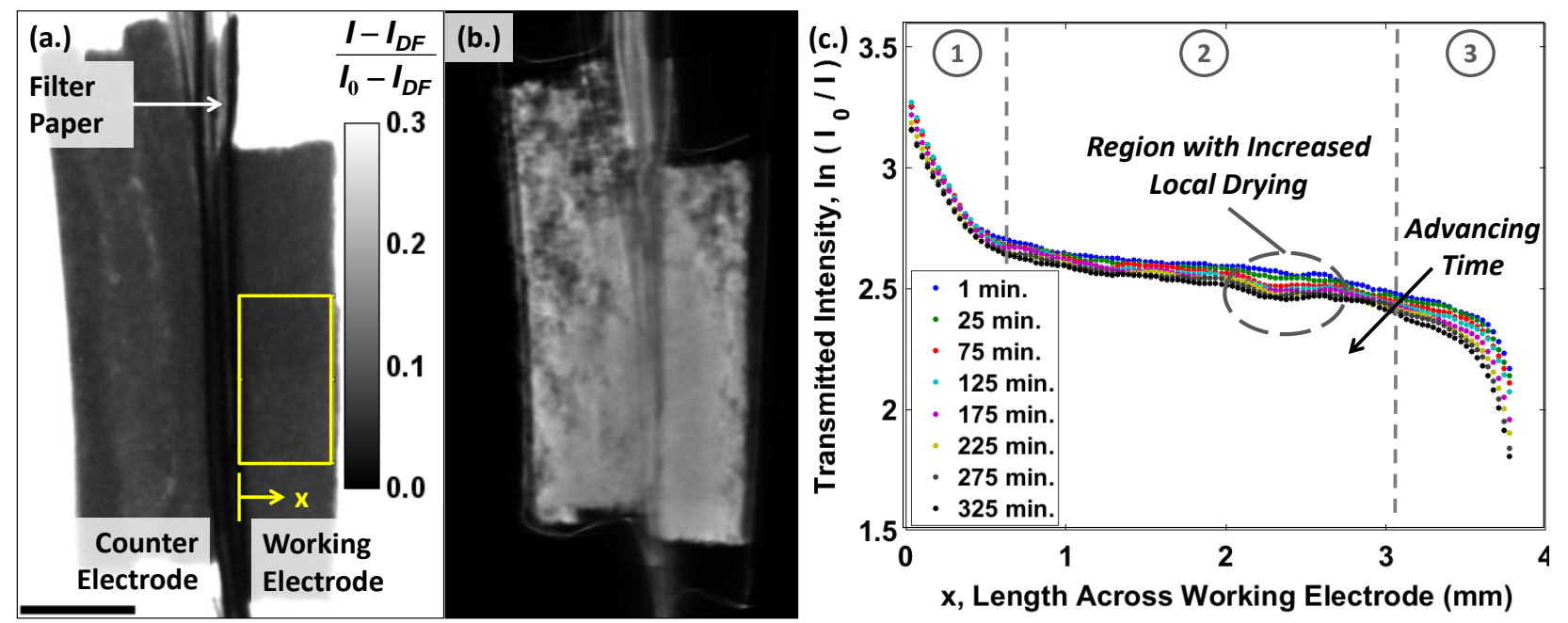

Figure 6. (a.) Transmission radiograph of test cell structure at time $t=60 \mathrm{~s}$. The image has been filtered to remove $\mathrm{Al}$ tape and current collectors. A region of interest with consistent saturation is indicated in yellow. A $5 \mathrm{~mm}$ scale bar is shown at the lower left. (b.) A crosssection of the EEC pouch cell taken from attenuation-based reconstructed tomographic data reveals regions where drying of the electrodes has occurred, as indicated by the dark regions within the working and counter electrodes. (c.) Relative absorption behavior indicates a gradual change in solution content due to combined effects of reaction and electrode drying. Three regions are seen corresponding to the active region (1), the bulk electrode (2), and the edge of the electrode near the current collector (3). 